\title{
Boolean Algebra of C-Algebras
}

\author{
G.C. Rao $^{1} \&$ P. Sundarayya ${ }^{2}$ \\ ${ }^{1}$ Department of Mathematics, Andhra University, Visakhapatnam-530 003, India \\ ${ }^{2}$ Department of Mathematics, GIT, GITAM University, Visakhapatnam-530 045, India \\ E-mails: gcraomaths@yahoo.co.in; psundarayya@yahoo.co.in
}

\begin{abstract}
A C-algebra is the algebraic form of the 3-valued conditional logic, which was introduced by F. Guzman and C.C. Squier in 1990. In this paper, some equivalent conditions for a $\mathrm{C}$ - algebra to become a boolean algebra in terms of congruences are given. It is proved that the set of all central elements $\mathrm{B}(\mathrm{A})$ is isomorphic to the Boolean algebra $\mathfrak{B}_{S(A)}$ of all C-algebras $\mathrm{S}_{\mathrm{a}}$, where a $\in \mathrm{B}(\mathrm{A})$. It is also proved that $\mathrm{B}(\mathrm{A})$ is isomorphic to the Boolean algebra $\mathfrak{B}_{R(A)}$ of all $C$-algebras $A_{a}$, where $a \in B(A)$.
\end{abstract}

Keywords: Boolean algebra; C-algebra; central element; permutable congruences.

\section{Introduction}

The concept of C-algebra was introduced by Guzman and Squier as the variety generated by the 3 -element algebra $\mathrm{C}=\{\mathrm{T}, \mathrm{F}, \mathrm{U}\}$. They proved that the only subdirectly irreducible $\mathrm{C}$-algebras are either $\mathrm{C}$ or the 2-element Boolean algebra $\mathrm{B}=\{\mathrm{T}, \mathrm{F}\}[1,2]$.

For any universal algebra $\mathrm{A}$, the set of all congruences on $\mathrm{A}$ (denoted by $\operatorname{Con} \mathrm{A}$ ) is a lattice with respect to set inclusion. We say that the congruences $\theta, \phi$ are permutable if $\theta \circ \varphi=\varphi \circ \theta$. We say that $\operatorname{Con} A$ is permutable if $\theta \circ \varphi=\varphi \circ \theta$ for all $\theta, \phi \in \operatorname{Con} A$. It is known that $\operatorname{Con} A$ need not be permutable for any $\mathrm{C}$ algebra $\mathrm{A}$.

In this paper, we give sufficient conditions for congruences on a $\mathrm{C}$-algebra $\mathrm{A}$ to be permutable. Also we derive necessary and sufficient conditions for a Calgebra $\mathrm{A}$ to become a Boolean algebra in terms of the congruences on $\mathrm{A}$. We also prove that the three Boolean algebras $\mathrm{B}(\mathrm{A})$, the set of $\mathrm{C}$-algebras $\mathfrak{B}_{S(A)}$ and the set of C-algebras $\mathfrak{B}_{R(A)}$ are isomorphic to each other.

Received November $1^{\text {st }}, 2011$, Revised April $5^{\text {th }}, 2012$, Accepted for publication July $7^{\text {th }}, 2012$

Copyright (C) 2012 Published by LPPM ITB, ISSN: 1978-3043, DOI: 10.5614/itbj.sci.2012.44.3.1 


\section{Preliminaries}

In this section we recall the definition of a $\mathrm{C}$-algebra and some results from $[1,3,5]$ which will be required later.

Definition 2.1. By a $C$-algebra we mean an algebra $\left\langle A, \wedge, \vee,{ }^{\prime}\right\rangle$ of type $(2,2,1)$ satisfying the following identities [1].
(a) $x^{\prime \prime}=x$;
(b) $(x \wedge y)^{\prime}=x^{\prime} \vee y^{\prime}$
(c) $x \wedge(y \wedge z)=(x \wedge y) \wedge z$
(d) $x \wedge(y \vee z)=(x \wedge y) \vee(x \wedge z)$
(e) $(x \vee y) \wedge z=(x \wedge z) \vee\left(x^{\prime} \wedge y \wedge z\right)$;
(f) $x \vee(x \wedge y)=x$
(g) $(x \wedge y) \vee(y \wedge x)=(y \wedge x) \vee(x \wedge y)$.

\section{Example 2.2. [1]:}

The 3- element algebra $\mathrm{C}=\{\mathrm{T}, \mathrm{F}, \mathrm{U}\}$ is a $\mathrm{C}$-algebra with the operations $\wedge, \vee$ and ' defined as in the following tables.

\begin{tabular}{c|c}
$x$ & $x^{\prime}$ \\
\hline $\mathrm{T}$ & $\mathrm{F}$ \\
$\mathrm{F}$ & $\mathrm{T}$ \\
$\mathrm{U}$ & $\mathrm{U}$
\end{tabular}

\begin{tabular}{c|ccc}
$\wedge$ & $\mathrm{T}$ & $\mathrm{F}$ & $\mathrm{U}$ \\
\hline $\mathrm{T}$ & $\mathrm{T}$ & $\mathrm{F}$ & $\mathrm{U}$ \\
$\mathrm{F}$ & $\mathrm{F}$ & $\mathrm{F}$ & $\mathrm{F}$ \\
$\mathrm{U}$ & $\mathrm{U}$ & $\mathrm{U}$ & $\mathrm{U}$
\end{tabular}

\begin{tabular}{c|ccc}
$\vee$ & $\mathrm{T}$ & $\mathrm{F}$ & $\mathrm{U}$ \\
\hline $\mathrm{T}$ & $\mathrm{T}$ & $\mathrm{T}$ & $\mathrm{T}$ \\
$\mathrm{F}$ & $\mathrm{T}$ & $\mathrm{F}$ & $\mathrm{U}$ \\
$\mathrm{U}$ & $\mathrm{U}$ & $\mathrm{U}$ & $\mathrm{U}$
\end{tabular}

Every Boolean algebra is a C-algebra.

Lemma 2.3. Every C-algebra satisfies the following laws [1,3,5].
(a) $x \wedge x=x$
(b) $x \wedge y=x \wedge\left(x^{\prime} \vee y\right)=\left(x^{\prime} \vee y\right) \wedge x$
(c) $x \vee\left(x^{\prime} \wedge x\right)=x$
(d) $\left(x \vee x^{\prime}\right) \wedge y=(x \wedge y) \vee\left(x^{\prime} \wedge y\right)$
(e) $\left(x \vee x^{\prime}\right) \wedge x=x$
(f) $x \vee x^{\prime}=x^{\prime} \vee x$
(g) $x \vee y \vee x=x \vee y$;
(h) $x \wedge x^{\prime} \wedge y=x \wedge x^{\prime}$;
(i) $x \wedge(y \vee x)=(x \wedge y) \vee x$.

The duals of all above statements are also true. 
Definition 2.4. If $A$ has identity $\mathrm{T}$ for $\wedge$ ( that is, $x \wedge T=T \wedge x=x$ for all $x \in A$ ), then it is unique and in this case, we say that $\mathrm{A}$ is a $C$-algebra with $\mathrm{T}$. We denote $T^{\prime}$ by $\mathrm{F}$ and this $\mathrm{F}$ is the identity for $\vee[1]$.

Lemma 2.5 [1]: Let A be a $C$-algebra with $\mathrm{T}$ and $x, y \in \mathrm{A}$. Then

(i) $x \vee y=F$ if and only if $x=y=F$

(ii) if $x \vee y=T$ then $x \vee x^{\prime}=T$.

(iii) $x \vee T=x \vee x^{\prime}$

(iv) $x \wedge F=x \wedge x^{\prime}$

Theorem 2.6. Let $\left\langle A, \wedge, \vee,{ }^{\prime}\right\rangle$ be a C-algebra. Then the following are equivalent [6]:

(i) A is a Boolean algebra.

(ii) $x \vee(y \wedge x)=x$, for all $x, y \in A$.

(iii) $x \wedge y=y \wedge x$, for all $x, y \in A$.

(iv) $(x \vee y) \wedge y=y$, for all $x, y \in A$.

(v) $\quad x \vee x^{\prime}$ is the identity for $\wedge$, for every $x \in A$.

(vi) $x \vee x^{\prime}=y \vee y^{\prime}$, for all $x, y \in A$.

(vii) A has a right zero for $\wedge$.

(viii) for any $x, y \in A$, there exists $a \in A$ such that $x \vee a=y \vee a=a$.

(ix) for any $x, y \in A$, if $x \vee y=y$, then $y \wedge x=x$.

Definition 2.7. Let $\mathrm{A}$ be a $\mathrm{C}$-algebra with $\mathrm{T}$. An element $x \in A$ is called a central element of A if $x \vee x^{\prime}=T$. The set of all central elements of A is called the Centre of $\mathrm{A}$ and is denoted by $\mathrm{B}(\mathrm{A})$ [5].

Theorem 2.8. Let $\mathrm{A}$ be a $\mathrm{C}$-algebra with $\mathrm{T}$.Then $\left\langle B(A), \wedge, \vee{ }^{\prime}\right\rangle$ is a Boolean Algebra [5].

\section{Some Properties of a $C$-algebra and Its Congruences}

In this section we prove some important properties of a $\mathrm{C}$-algebra and we give sufficient conditions for two congruences on a $\mathrm{C}$-algebra $\mathrm{A}$ to be permutable. Also we derive necessary and sufficient conditions for a $\mathrm{C}$-algebra $\mathrm{A}$ to become a Boolean algebra in terms of the congruences on A.

Lemma 3.1. Every C-algebra satisfies the following identities: 
(i) $x \vee y=x \vee\left(y \wedge x^{\prime}\right)$;

(ii) $x \wedge y=x \wedge\left(y \vee x^{\prime}\right)$.

Proof. Let A be a C-algebra and $x, y \in A$. Now,

$x \vee y=x \vee\left(x^{\prime} \wedge y\right)=x \vee\left(x^{\prime} \wedge y \wedge x^{\prime}\right)=\left[x \wedge\left(x \vee x^{\prime}\right)\right] \vee\left[x^{\prime} \wedge y \wedge\left(x^{\prime} \wedge\left(x \vee x^{\prime}\right)\right)\right]=$ $\left[x \wedge\left(x \vee x^{\prime}\right)\right] \vee\left[x^{\prime} \wedge y \wedge x^{\prime} \wedge\left(x \vee x^{\prime}\right)\right]=\left[x \wedge\left(x \vee x^{\prime}\right)\right] \vee\left[x^{\prime} \wedge y \wedge\left(x \vee x^{\prime}\right)\right]=(x \vee y)$ $\wedge\left(x \vee x^{\prime}\right)=x \vee\left(y \wedge x^{\prime}\right)$. Similarly $x \wedge y=x \wedge\left(y \vee x^{\prime}\right)$.

Lemma 3.2. Let A be a C-algebra and $x, y \in A$. Then $x \vee y \vee x^{\prime}=x \vee y \vee y^{\prime}$. Proof. Let A be a C-algebra and $x, y \in A$. By Lemma 2.3[b],[f] and Lemma 3.1, we have $x \vee y \vee x^{\prime}=x \vee\left(\left(y^{\prime} \wedge x^{\prime}\right) \vee y\right)=\left[x \vee\left(y^{\prime} \wedge x^{\prime}\right)\right] \vee y=\left(x \vee y^{\prime}\right) \vee$ $y=x \vee y^{\prime} \vee y=x \vee y \vee y^{\prime}$.

Lemma 3.3. Let $A$ be a C-algebra with $T, x, y \in A$ and $x \wedge y=F$. Then $x \vee y=y \vee x$.

Proof. Suppose that $x \wedge y=F$. Then $F=x \wedge y=x \wedge\left(x^{\prime} \vee y\right)=\left(x \wedge x^{\prime}\right) \vee$ $(x \wedge y)=\left(x \wedge x^{\prime}\right) \vee F=x \wedge x^{\prime}$. now $x \vee y=F \vee(x \vee y)=(x \wedge y) \vee(x \vee y)$ $=(x \vee x \vee y) \wedge\left(x^{\prime} \vee y \vee x \vee y\right)$ (By Def 2.1) $=(x \vee y) \wedge\left(x^{\prime} \vee y \vee x\right)$ (By $2.3[\mathrm{~g}])=\left(x \wedge x^{\prime}\right) \vee(y \vee x)=F \vee(y \vee x)=y \vee x$.

In [6], it is proved that if $\mathrm{A}$ is a C-algebra with $\mathrm{T}$ then $B(A)=\{a \in A \mid$ $\left.a \vee a^{\prime}=T\right\}$ is a Boolean algebra under the same operations $\wedge, \vee, '$ in the Calgebra A. Now we prove the following.

Theorem 3.4. Let $\mathrm{A}$ be a $\mathrm{C}$-algebra with $\mathrm{T}$ and $a, b \in A$ such that $a \vee b \in B(A)$. Then $a \in B(A)$.

Proof. Let A be a C-algebra with $\mathrm{T}$ and $a, b \in A$ such that $a \vee b \in B(A)$. Then $T=(a \vee b) \vee(a \vee b)^{\prime}=(a \vee b) \vee\left(a^{\prime} \wedge b^{\prime}\right)$

$=\left(a \vee b \vee a^{\prime}\right) \wedge\left(a \vee b \vee b^{\prime}\right)=\left(a \vee b \vee a^{\prime}\right) \wedge\left(a \vee b \vee a^{\prime}\right)$ (By Theorem 3.2)

$=a \vee b \vee a^{\prime}$.

Therefore, $T=a \vee b \vee a^{\prime}$ 


$$
\text { Now, } \begin{aligned}
a \vee a^{\prime} & =\left(a \vee a^{\prime}\right) \wedge T \\
& =\left(a \vee a^{\prime}\right) \wedge\left(a \vee b \vee a^{\prime}\right) \quad(\mathrm{by}(\mathrm{I})) \\
& =\left(a \wedge\left(a \vee b \vee a^{\prime}\right)\right) \vee\left(a^{\prime} \wedge\left(a \vee b \vee a^{\prime}\right)\right) \\
& =a \vee\left(a^{\prime} \wedge\left(a \vee b \vee a^{\prime}\right)\right) \\
& =a \vee\left(a \vee b \vee a^{\prime}\right) \quad(\text { By 2.3[b]) } \\
& =a \vee b \vee a^{\prime}=T .
\end{aligned}
$$

Hence $a \in B(A)$.

The converse of the above theorem need not be true. For example, in the Calgebra $C, F \in B(C)$ but $F \vee U=U \notin B(C)$. We have the following consequence of the above theorem.

Corollary 3.5. Let A be a C-algebra with $\mathrm{T}, a, b \in A$ and $a \wedge b \in B(A)$. Then $a \in B(A)$.

Proof. Let $a \wedge b \in B(A)$. Then we have, $(a \wedge b)^{\prime} \in B(A) \Rightarrow a^{\prime} \vee b^{\prime} \in B(A)$ $\Rightarrow a^{\prime} \in B(A) \Rightarrow a \in B(A)$.

In [1], it is proved that if $\mathrm{A}$ is a $\mathrm{C}$-algebra, then $\theta_{x}=\{(p, q) \mid x \wedge p=x \wedge q\}$ is a congruence on A and $\theta_{x} \cap \theta_{x^{\prime}}=\theta_{x \vee x^{\prime}}$. In [6], if A is C-algebra with $\mathrm{T}$ then $\theta_{x}$ is a factor congruence if and only if $x \in B(A)$. They also proved that $\theta_{x}, \theta_{y}$ are permutable congruences whenever both $x, y \in B(A)$. Now we prove some important properties of these congruences.

Theorem 3.6. Let $\mathrm{A}$ be a $\mathrm{C}$-algebra with $\mathrm{T}$ and $a, b \in A$. Then we have the following (i) $\theta_{a \wedge b}=\theta_{b \wedge a}$; (ii) $\theta_{a} \circ \theta_{b} \subseteq \theta_{a \wedge b}$.

Proof. (i) $(x, y) \in \theta_{a \wedge b}$

$$
\begin{aligned}
& \Rightarrow a \wedge b \wedge x=a \wedge b \wedge y \\
& \Rightarrow b \wedge a \wedge b \wedge x=b \wedge a \wedge b \wedge x \\
& \Rightarrow b \wedge a \wedge x=b \wedge a \wedge x \\
& \Rightarrow(x, y) \in \theta_{b \wedge a}
\end{aligned}
$$

Therefore $\theta_{a \wedge b} \subseteq \theta_{b \wedge a}$. Similarly, $\theta_{b \wedge a} \subseteq \theta_{a \wedge b}$. Hence $\theta_{a \wedge b}=\theta_{b \wedge a}$. 
(ii) Let $(x, y) \in \theta_{a} \circ \theta_{b}$. Then there exists $z \in A$ such that $(x, z) \in \theta_{b}$ and $(z, y) \in \theta_{a}$. Thus $b \wedge x=b \wedge z$ and $a \wedge z=a \wedge y$. Now, $a \wedge b \wedge x=a \wedge b \wedge$ $z=a \wedge b \wedge a \wedge z=a \wedge b \wedge a \wedge y=a \wedge b \wedge y$. Therefore, $(x, y) \in \theta_{a \wedge b}$. Thus $\theta_{a} \circ \theta_{b} \subseteq \theta_{a \wedge b}$.

In the following we give an example of a $\mathrm{C}$-algebra $\mathrm{G}$ without $\mathrm{T}$ in which the Con $\mathrm{A}$ is not permute.

Example 3.7. Consider the C-algebra $G=\left\{a_{1}, a_{2}, a_{3}, a_{4}, a_{5}\right\}$ where $a_{1}=(T, U)$, $a_{2}=(F, U), a_{3}=(U, T), a_{4}=(U, F), a_{5}=(U, U)$ under pointwise operations in $\mathrm{C}$.

\begin{tabular}{|l|l|}
\hline$x$ & $x^{\prime}$ \\
\hline$a_{1}$ & $a_{2}$ \\
\hline$a_{2}$ & $a_{1}$ \\
\hline$a_{3}$ & $a_{4}$ \\
\hline$a_{4}$ & $a_{3}$ \\
\hline$a_{5}$ & $a_{5}$ \\
\hline
\end{tabular}

\begin{tabular}{|c|c|c|c|c|c|}
\hline$\wedge$ & $a_{1}$ & $a_{2}$ & $a_{3}$ & $a_{4}$ & $a_{5}$ \\
\hline$a_{1}$ & $a_{1}$ & $a_{2}$ & $a_{5}$ & $a_{5}$ & $a_{5}$ \\
\hline$a_{2}$ & $a_{2}$ & $a_{2}$ & $a_{2}$ & $a_{2}$ & $a_{2}$ \\
\hline$a_{3}$ & $a_{5}$ & $a_{5}$ & $a_{3}$ & $a_{4}$ & $a_{5}$ \\
\hline$a_{4}$ & $a_{4}$ & $a_{4}$ & $a_{4}$ & $a_{4}$ & $a_{4}$ \\
\hline$a_{5}$ & $a_{5}$ & $a_{5}$ & $a_{5}$ & $a_{5}$ & $a_{5}$ \\
\hline
\end{tabular}

\begin{tabular}{|c|c|c|c|c|c|}
\hline$\vee$ & $a_{1}$ & $a_{2}$ & $a_{3}$ & $a_{4}$ & $a_{5}$ \\
\hline$a_{1}$ & $a_{1}$ & $a_{1}$ & $a_{1}$ & $a_{1}$ & $a_{1}$ \\
\hline$a_{2}$ & $a_{1}$ & $a_{2}$ & $a_{5}$ & $a_{5}$ & $a_{5}$ \\
\hline$a_{3}$ & $a_{3}$ & $a_{3}$ & $a_{3}$ & $a_{3}$ & $a_{3}$ \\
\hline$a_{4}$ & $a_{5}$ & $a_{5}$ & $a_{3}$ & $a_{4}$ & $a_{5}$ \\
\hline$a_{5}$ & $a_{5}$ & $a_{5}$ & $a_{5}$ & $a_{5}$ & $a_{5}$ \\
\hline
\end{tabular}

This algebra $\left(G, \vee, \wedge,,^{\prime}\right)$ is a $\mathrm{C}$-algebra with out $\mathrm{T}$.

Let $\Delta=$ diagonal of A. Then we have the following:

$$
\begin{aligned}
\theta_{a_{1}} & =\left\{(x, y) \mid a_{1} \wedge x=a_{1} \wedge y\right\} \\
& =\Delta \cup\left\{\left(a_{3}, a_{4}\right),\left(a_{4}, a_{5}\right),\left(a_{5}, a_{3}\right),\left(a_{4}, a_{3}\right)\left(a_{5}, a_{4}\right),\left(a_{3}, a_{5}\right)\right\} \\
\theta_{a_{3}} & =\Delta \cup\left\{\left(a_{1}, a_{2}\right),\left(a_{2}, a_{5}\right),\left(a_{5}, a_{1}\right),\left(a_{2}, a_{1}\right)\left(a_{5}, a_{2}\right),\left(a_{1}, a_{5}\right)\right\}
\end{aligned}
$$


Now, $\quad \theta_{a_{1}} \circ \theta_{a_{3}}=\Delta \cup \theta_{a_{1}} \cup \theta_{a_{3}} \cup\left\{\left(a_{4}, a_{1}\right),\left(a_{4}, a_{2}\right)\left(a_{3}, a_{1}\right),\left(a_{3}, a_{2}\right)\right\}$

$$
\theta_{a_{3}} \circ \theta_{a_{1}}=\Delta \cup \theta_{a_{1}} \cup \theta_{a_{3}} \cup\left\{\left(a_{2}, a_{4}\right),\left(a_{2}, a_{3}\right)\left(a_{1}, a_{3}\right),\left(a_{1}, a_{4}\right)\right\}
$$

Therefore $\theta_{a_{1}} \circ \theta_{a_{3}} \neq \theta_{a_{3}} \circ \theta_{a_{1}}$.

Theorem 3.8. Let $\mathrm{A}$ a $\mathrm{C}$-algebra with $\mathrm{T}$ and $a \in B(A)$. Then for any $b \in A, \theta_{a}, \theta_{b}$ permute and $\theta_{a} \circ \theta_{b}=\theta_{a \wedge b}$.

Proof. Let $\mathrm{A}$ be a C-algebra with $\mathrm{T}$ and $a \in B(A)$. By Theorem 3.6, $\theta_{a} \circ \theta_{b} \subseteq \theta_{a \wedge b}$. Now let $(p, q) \in \theta_{a \wedge b}$. Then $a \wedge b \wedge p=a \wedge b \wedge q \Rightarrow b \wedge a \wedge b$ $\wedge p=b \wedge a \wedge b \wedge q \Rightarrow b \wedge a \wedge p=b \wedge a \wedge q$. Consider, $r=(a \wedge p) \vee\left(a^{\prime} \wedge q\right)$. Now $a \wedge r=a \wedge\left[(a \wedge p) \vee\left(a^{\prime} \wedge q\right)\right]=(a \wedge p) \vee\left(a \wedge a^{\prime} \wedge q\right)=(a \wedge p) \vee(F \wedge q)$ $=(a \wedge p) \vee F=a \wedge p$. Therefore $(r, p) \in \theta_{a} \Rightarrow(p, r) \in \theta_{a}$. Now, $b \wedge r=$ $b \wedge\left[(a \wedge p) \vee\left(a^{\prime} \wedge q\right)\right]=[b \wedge a \wedge p] \vee\left[b \wedge a^{\prime} \wedge q\right]=(b \wedge a \wedge q) \vee\left(b \wedge a^{\prime} \wedge q\right)$ $=b \wedge\left((a \wedge q) \vee\left(a^{\prime} \wedge q\right)\right)=b \wedge\left(\left(a \vee a^{\prime}\right) \wedge q\right)=b \wedge(T \wedge q)$ (since $\left.a \in B(A)\right)$ $=b \wedge q$. Therefore $(q, r) \in \theta_{b} \Rightarrow(r, q) \in \theta_{b}$. Thus $(p, q) \in \theta_{b} \circ \theta_{a}$. Hence $\theta_{b} \circ \theta_{a}=\theta_{a \wedge b}$. Thus $\theta_{b} \circ \theta_{a}$ is a congruence on $\mathrm{A}$ and hence $\theta_{a}, \theta_{b}$ are permutable congruences and hence $\theta_{a} \circ \theta_{b}=\theta_{b} \circ \theta_{a}=\theta_{a \wedge b}$.

Corollary 3.9. Let $\mathrm{A}$ be a $\mathrm{C}$-algebra with $\mathrm{T}$ and $a, b \in A$. Then i) $a \vee b \in B(A) \Rightarrow \theta_{a} \circ \theta_{b}=\theta_{a \wedge b}$; ii) $a \wedge b \in B(A) \Rightarrow \theta_{a} \circ \theta_{b}=\theta_{a \wedge b}$.

Proof. i) We know that if $a \vee b \in B(A)$ then $a \in B(A)$ and hence by the above theorem $\theta_{a} \circ \theta_{b}=\theta_{b} \circ \theta_{a}=\theta_{a \wedge b}$. Similarly, we can prove ii).

Let $\mathrm{A}$ be a $\mathrm{C}$-algebra. If Con( $\mathrm{A})$ is permutable, then $\mathrm{A}$ need not be a Boolean algebra. For example, in the $\mathrm{C}$-algebra $\mathrm{C}$, the only congruences are $\Delta, \nabla$ and they are permutable. But $\mathrm{C}$ is not a Boolean algebra. Now we give equivalent conditions for a $\mathrm{C}$-algebra to become a Boolean algebra in terms of congruence relations.

Theorem 3.10. Let $\left(A, \vee, \wedge,,^{\prime}\right)$ be a C-algebra with $\mathrm{T}$. Then the following are equivalent. (i) Let $\left(A, \vee, \wedge,{ }^{\prime}\right)$ be a Boolean algebra. (ii) $\theta_{x} \cap \theta_{x^{\prime}}=\Delta$ for all $x \in A$. (iii) $\theta_{x \vee x^{\prime}}=\Delta$ for all $x \in A$. 
Proof. (1) $\Rightarrow(2)$ : Let A be a Boolean algebra and $x \in A$. Let $(p, q) \in \theta_{x} \cap \theta_{x^{\prime}}$. Then $x \wedge p=x \wedge q$ and $x^{\prime} \wedge p=x^{\prime} \wedge q$. Now, $p=\left(x \vee x^{\prime}\right) \wedge p=(x \wedge p) \vee$ $\left(x^{\prime} \wedge q\right)=(x \wedge q) \vee\left(x^{\prime} \wedge q\right)=\left(x \vee x^{\prime}\right) \wedge q=q$. Thus $\theta_{x} \cap \theta_{x^{\prime}} \subseteq \Delta$. Therefore $\theta_{x} \cap \theta_{x^{\prime}}=\Delta$. Since $\theta_{x} \cap \theta_{x^{\prime}}=\theta_{x \vee x^{\prime}}$, we get (ii) $\Rightarrow$ (iii). (iii) $\Rightarrow$ (i): Suppose $\theta_{x \vee x^{\prime}}=\Delta$ for all $x \in A$. We prove that $\theta_{x^{\prime}} \circ \theta_{x}=A \times A$. Let $(p, q) \in A \times A$. Write $t=(x \wedge p) \vee\left(x^{\prime} \wedge q\right)$. Now, $x \wedge t=x \wedge\left((x \wedge p) \vee\left(x^{\prime} \wedge q\right)\right)=(x \wedge p)$ $\vee\left(x \wedge x^{\prime} \wedge q\right)=(x \wedge p) \vee\left(x \wedge x^{\prime}\right)=x \wedge\left(p \vee x^{\prime}\right)=x \wedge p$. Also, $x^{\prime} \wedge t=x^{\prime} \wedge$ $\left.\left.\left((x \wedge p) \vee\left(x^{\prime} \wedge q\right)\right)=\left(x^{\prime} \wedge x \wedge p\right) \vee\left(x^{\prime} \wedge x^{\prime} \wedge q\right)\right)=\left(x^{\prime} \wedge x\right) \vee\left(x^{\prime} \wedge q\right)\right)=\left(x^{\prime} \wedge(x\right.$ $\vee q))=x^{\prime} \wedge q$. Therefore $(p, t) \in \theta_{x}$ and $(t, q) \in \theta_{x^{\prime}}$. Thus $(p, q) \in \theta_{x^{\prime}} \circ \theta_{x}$. Hence we get $\theta_{x^{\prime}} \circ \theta_{x}=A \times A$. Also $\theta_{x} \cap \theta_{x^{\prime}}=\theta_{x \vee x^{\prime}}=\Delta$. That is $\theta_{x}$ and $\theta_{x^{\prime}}$ are permutable factor congruences. Therefore, by Theorem 2.6, we have $x \in B(A)$. Thus $A=B(A)$ and hence $\mathrm{A}$ is a Boolean algebra.

\section{The C-algebra $S_{x}$}

We prove that, for each $x \in A, S_{x}=\{x \vee t \mid t \in A\}$ is itself a C-algebra under induced operations $\wedge, \vee$ and the unary operation is defines by $(x \vee t)^{*}=x \wedge t^{\prime}$ . We observe that $S_{\mathrm{x}}$ need not be a subalgebra of $A$ because the unary operation in $\mathrm{S}_{\mathrm{x}}$ is not the restriction of the unary operation on A. Also for each $x \in A$, the set $A_{x}=\{x \wedge t \mid t \in A\}$ is a C-algebra in which the unary operation is given by $(x \wedge t)^{*}=x \wedge t^{\prime}$. We prove that the $B(A)$ is isomorphic to the Boolean algebra $\mathfrak{B}_{S(A)}$ of all C-algebras $\mathrm{S}_{\mathrm{a}}$ where $a \in B(A)$. Also, we prove that $\mathrm{B}(\mathrm{A})$ is isomorphic to the Boolean algebra $\mathfrak{B}_{R(A)}$ of all C-algebras $A_{a}, a \in B(A)$.

Theorem 4.1. Let $\left\langle A, \wedge, \vee,^{\prime}\right\rangle$ be a C-algebra, $x \in A$ and $S_{x}=\{x \vee t \mid t \in A\}$. Then $\left\langle S_{x}, \wedge, \vee, *\right\rangle$ is a C-algebra with $x$ as the identity for $\vee$, where $\wedge$ and $\vee$ are the operations in A restricted to $\mathrm{S}_{\mathrm{x}}$ and for any $x \vee t \in S_{x}$, here $(x \vee t)^{*}$ is $x \vee t^{\prime}$.

Proof. Let $t, r, s \in A$. Then $(x \vee t) \vee(x \vee r)=x \vee(t \vee r) \in S_{x}$ and $(x \vee t) \wedge$ $(x \vee r)=x \wedge(t \vee r) \in S_{x}$. Thus $\vee, \wedge$ are closed in $\mathrm{S}_{\mathrm{x}}$. Also ${ }^{*}$ is closed in $\mathrm{S}_{\mathrm{x}}$. Consider $(x \vee t)^{* * *}=x \vee\left(x \vee t^{\prime}\right)^{\prime}=x \vee\left(x^{\prime} \wedge t\right)=x \vee t$. Now $[(x \vee t) \wedge(x \vee r)]^{*}$ 
$=[x \vee(t \wedge r)]^{*}=x \vee\left(t^{\prime} \vee r^{\prime}\right)=x \vee t^{\prime} \vee x \vee r^{\prime}=(x \vee t)^{*} \vee(x \vee r)^{*} . \quad$ Now, consider $\quad(x \vee t) \vee(x \vee r) \wedge(x \vee s)=x \wedge[(t \wedge r) \wedge s]=x \vee(t \wedge s) \vee\left(t^{\prime} \wedge r \wedge s\right)$ $=x \vee(t \wedge s) \vee x \vee\left(t^{\prime} \wedge r \wedge s\right)=(x \vee t) \wedge(x \vee s) \vee\left(x \vee t^{\prime}\right) \wedge(x \vee r) \wedge(x \vee s)=$ $(x \vee t) \wedge(x \vee s) \underset{\vee}{\vee}(x \vee t)^{*} \wedge(x \vee r) \wedge(x \vee s)$. The remaining identities of a $\mathrm{C}$-algebra also hold in $\mathrm{S}_{\mathrm{x}}$ because they hold in A. Hence, $\mathrm{S}_{\mathrm{x}}$ is itself a C-algebra. Also $x$ is the identity for $\vee$ because $x \vee x \vee t=x \vee t=x \vee t \vee x$. Here $x \vee x^{\prime}$ is the identity for $\wedge$.

Theorem 4.2. Let A be a C-algebra. Then the following holds.

(i) $S_{x}=S_{y}$ if and only if $x=y$;

(ii) $S_{x} \cap S_{y} \subseteq S_{x \vee y}$;

(iii) $S_{x} \cap S_{x^{\prime}}=S_{x \vee x^{\prime}}$;

(iv) $\left(S_{x}\right)_{x \vee y}=S_{x \vee y}$.

Proof. (i) Suppose $S_{x}=S_{y}$. Since $x=x \vee x \in S_{x}=S_{y}$ and $y=y \vee y \in$ $S_{y}=S_{x}$. Therefore $x=y \vee t$ and $y=x \vee r$ for some $t, r \in A$. Now, $x=y \vee t=(y \vee t \vee y) \wedge(y \vee y \vee t)=(x \vee y) \wedge(y \vee x)=(y \vee x) \wedge(x \vee y)=$ $(x \vee r \vee x) \wedge(x \vee x \vee r)=x \vee r=y$. The converse is trivial. (ii) Suppose $t \in S_{x} \cap S_{y}$. Then $t=x \vee s=y \vee r$ for some $s, r \in A$. Now, $t=x \vee x \vee s$ $=x \vee t=x \vee y \vee r \in S_{x \vee y}$. (iii) $S_{x} \cap S_{x^{\prime}} \subseteq S_{x \vee x^{\prime}}$ by (ii). Since $x \vee x^{\prime}=x^{\prime} \vee x$ we have $S_{x \vee x^{\prime}} \subseteq S_{x} \cap S_{x^{\prime}}$. Hence $S_{x} \cap S_{x^{\prime}}=S_{x \vee x^{\prime}}$. (iv) $\left(S_{x}\right)_{x \vee y}=\{x \vee y \vee t \mid$ $\left.t \in S_{x}\right\}=\{x \vee y \vee x \vee r \mid r \in A\}=\{x \vee y \vee r \mid r \in A\}=S_{x \vee y}$.

Theorem 4.3. Let $\mathrm{A}$ be a $\mathrm{C}$-algebra with $\mathrm{T}$ and $x \in A$, then the mapping $\alpha_{x}$ : $A \rightarrow S_{x}$ defined by $\alpha_{x}(t)=x \vee t$ for all $t \in A$ is a homomorphism of A to $S_{x}$ with kernel $\theta_{x^{\prime}}$ and hence $A / \theta_{x^{\prime}} \cong S_{x}$.

Proof. Let $t, r \in A$. Then $\alpha_{x}(t \vee r)=x \vee t \vee r=x \vee t \vee x \vee r=\alpha_{x}(t) \vee \alpha_{x}(r)$ and $\alpha_{x}\left(t^{\prime}\right)=x \vee t^{\prime}=(x \vee t)^{*}=\left(\alpha_{x}(t)\right)^{*}$. Clearly, $\alpha_{x}(t \wedge r)=\alpha_{x}(t) \wedge \alpha_{x}(r)$. Also $\alpha_{x}(T)=x \vee T=x \vee x^{\prime}$, which is the identity for $\wedge$ in $S_{x}$. Therefore $\alpha_{x}$ is a homomorphism. Hence by the fundamental theorem of homomorphism $A / \operatorname{Ker}_{x} \cong S_{x}$ and $\operatorname{Ker}_{x}=\left\{(t, r) \in A \times A \mid \alpha_{x}(t)=\alpha_{x}(r)\right\}=\{(t, r) \in A \times A \mid$ 
$x \vee t=x \vee r\}=\left\{(t, r) \in A \times A \mid x^{\prime} \wedge t=x^{\prime} \wedge r\right\} \theta_{x^{\prime}}$ (by Lemma $\left.2.3[\mathrm{~b}]\right)=$ and hence $A / \theta_{x^{\prime}} \cong S_{x}$.

Theorem 4.4. Let A be a C-algebra with T and $a \in B(A)$, then $A \cong S_{a} \times S_{a^{\prime}}$.

Proof. Define $\alpha: A \rightarrow S_{a} \times S_{a^{\prime}}$ by $\alpha(x)=\left(\alpha_{a}(x), \alpha_{a^{\prime}}(x)\right)$ for all $x \in A$. Then, by Theorem 4.3, $\alpha$ is well-defined and $\alpha$ is a homomorphism. Now, we prove that $\alpha$ is one-one. Let $x, y \in A$. Then $\alpha(x)=\alpha(y) \Rightarrow\left(\alpha_{a}(x), \alpha_{a^{\prime}}(x)\right)$ $=\left(\alpha_{a}(y), \alpha_{a^{\prime}}(y)\right) \Rightarrow\left(a \vee x, a^{\prime} \vee x\right)=\left(a \vee y, a^{\prime} \vee y\right) \Rightarrow a \vee x=a \vee y \quad$ and $a^{\prime} \vee x=a^{\prime} \vee y$. Now $x=F \vee x=\left(a \wedge a^{\prime}\right) \vee x=(a \vee x) \wedge\left(a^{\prime} \vee x\right)=(a \vee y) \wedge$ $\left(a^{\prime} \vee y\right)=y$. Finally, we prove that $\alpha$ is onto. Let $(x, y) \in S_{a} \times S_{a^{\prime}}$. Then $x=a \vee t$, and $y=a^{\prime} \vee r$ for some $t, r \in A$. Therefore, $a \vee x=x$, $a \vee y=a \vee a^{\prime} \vee y=T \vee y=T \quad$ and $\quad a^{\prime} \vee x=T, a^{\prime} \vee y=y$. Now, $\alpha(x \wedge y)=\left(\alpha_{a}(x \wedge y), \alpha_{a^{\prime}}(x \wedge y)\right)$ $=\left(a \vee(x \wedge y), a^{\prime} \vee(x \wedge y)\right)$ $=\left((a \vee x) \wedge(a \vee y),\left(a^{\prime} \vee x\right) \wedge\left(a^{\prime} \vee y\right)\right)$ $=(x \wedge T, T \wedge y)$ $=(x, y)$.

Therefore, $\alpha$ is onto and hence $\alpha$ is an isomorphism. Therefore $A \cong S_{a} \times S_{a^{\prime}}$.

Lemma 4.5. Let A be a C-algebra. Then for $a, b \in A$ :

(i) $a \vee b=b \vee a$ if and only if $S_{a \vee b}=S_{a} \cap S_{b}$

(ii) $S_{a \wedge b}=\operatorname{Sup}\left\{S_{a}, S_{b}\right\}$ in the poset $\left(\left\{S_{x} \mid x \in A\right\}, \subseteq\right)$, then $a \wedge b=b \wedge a$. The converse is not true.

Proof. (i) Suppose that $a \vee b=b \vee a$. Then clearly $S_{a \vee b} \subseteq S_{a} \cap S_{b}$. By Theorem 4.2(ii) $S_{a} \cap S_{b} \subseteq S_{a \vee b}$. Hence $S_{a \vee b}=S_{a} \cap S_{b}$. Conversely assume that $S_{a \vee b}=S_{a} \cap S_{b}$. Clearly $a \vee b \in S_{a \vee b}=S_{a} \cap S_{b}$. Therefore $a \vee b \in S_{b} \Rightarrow$ $a \vee b=b \vee t$ for some $t \in A$. Now $b \vee a=b \vee a \vee b=b \vee b \vee t=b \vee t=$ $a \vee b$. (ii) Assume that $a, b \in A$ and $S_{a \wedge b}=\operatorname{Sup}\left\{S_{a}, S_{b}\right\}$. Then $S_{a \wedge b}=S_{b \wedge a}$ and hence $a \wedge b \in S_{a \wedge b}=S_{b \wedge a}$. Therefore $a \wedge b=(b \wedge a) \vee t$ for some $t \in A$. 
Now $(b \wedge a) \vee(a \wedge b)=(b \wedge a) \vee((b \wedge a) \vee t)=(b \wedge a) \vee t=a \wedge b$. Similarly we can prove that $(a \wedge b) \vee(b \wedge a)=b \wedge a$. Hence $a \wedge b=b \wedge a$. The converse need not be true, for example for the C-algebra $C$, $S_{U}=\{U\}, S_{T}=\{T\}$ and $U \wedge T=T \wedge U$. But $S_{U \wedge T}\left(=S_{U}\right)$ is not an upper bound of $\left\{S_{U}, S_{T}\right\}$.

Now we prove $\mathfrak{B}_{S(A)}=\left\{S_{a} \mid a \in B(A)\right\}$ is a Boolean algebra under set inclusion.

Theorem 4.6. Let $\left\langle A, \wedge, \vee,^{\prime}\right\rangle$ be a C-algebra with $\mathrm{T}$. Then $\mathfrak{B}_{S(A)}=\left\{S_{a} \mid a \in B(A)\right\}$ is a Boolean algebra under set inclusion.

Proof. Clearly $\left(\mathfrak{B}_{S(A)}, \subseteq\right)$ is a partially ordered set under inclusion. First we show for $a, b \in B(A), S_{a \vee b}$ is the infimum of $\left\{S_{a}, S_{b}\right\}$ and $S_{a \wedge b}$ is the supremum of $\left\{S_{a}, S_{b}\right\}$ for all $a, b \in B(A)$. Let $a, b \in B(A)$. Then $a \wedge b=b \wedge a$ and $a \vee b=b \vee a$. Hence by the above Lemma 4.5, $S_{a \vee b}$ is the infimum of $\left\{S_{a}, S_{b}\right\}$. Let $t \in S_{a}$. Then $t=a \vee x$ for some $x \in A$. Now $t=a \vee x=(a \wedge(a \vee b)) \vee x=\left(a \wedge(b \vee a) \vee x=(a \wedge b) \vee a \vee x \in S_{a \wedge b}\right.$. Similarly $S_{b} \subseteq S_{b \wedge a}=S_{a \wedge b}$. Therefore $S_{a \wedge b}$ is an upper bound of $S_{a}, S_{b}$. Suppose $S_{c}$ is an upper bound of $S_{a}, S_{b} . t \in S_{a \wedge b}$. Then $t=(a \wedge b) \vee x$ for some $x \in A$. Now $t=(a \wedge b) \vee x=(a \vee x) \wedge\left(a^{\prime} \vee b \vee x\right)=(a \vee x) \wedge\left(b \vee a^{\prime} \vee x\right) \in S_{c}$ (since $a \vee x \in S_{a} \subseteq S_{c}, b \vee a^{\prime} \vee x \in S_{b} \subseteq S_{c}$ and $S_{c}$ is closed under $\left.\wedge\right)$. Therefore $S_{a \wedge b}$ is the supremum of $\left\{S_{a}, S_{b}\right\}$. Denote the supremum of $\left\{S_{a}, S_{b}\right\}$ by $S_{a} \vee S_{b}$ and the infimum of $\left\{S_{a}, S_{b}\right\}$ by $S_{a} \wedge S_{b}$. Now $S_{T} \wedge S_{a}=S_{T \vee a}=S_{T}$ and $S_{F} \vee S_{a}=S_{F \wedge a}=S_{F}$. Therefore $S_{T}$ is the least element and $S_{F}$ is the greatest element of $\left(\mathfrak{B}_{S(A)}, \subseteq\right)$. Now for any $a, b, c \in B(A),\left(S_{a} \vee S_{b}\right) \wedge S_{c}=S_{(a \wedge b) \vee c}=S_{(a \vee c \wedge \wedge(b \vee c)}=S_{(a \vee c)} \vee S_{(b \vee c)}=\left(S_{a} \wedge\right.$ $\left.S_{c}\right) \vee\left(S_{b} \wedge S_{c}\right)$. Also, $S_{a} \wedge S_{a^{\prime}}=S_{a \vee a^{\prime}}=S_{T} \quad$ and $\quad S_{a} \vee S_{a^{\prime}}=S_{a \wedge a^{\prime}}=S_{F}$. Therefore $\left(\mathfrak{B}_{S(A)}, \subseteq\right)$ is a complimented distributive lattice and hence it is a Boolean algebra. 
Theorem 4.7. Let $\mathrm{A}$ be a C-algebra with $\mathrm{T}$ Define $\varphi: B(A) \rightarrow \mathfrak{B}_{S(A)}$ by $\phi(a)=s_{a^{\prime}}$ for all $a \in B(A)$. Then $\phi$ is an isomorphism.

Proof. Let $a, b \in B(A)$. Then $\varphi(a \wedge b)=S_{(a \wedge b)^{\prime}}=S_{a^{\prime}} \wedge S_{b^{\prime}}=\varphi(a) \wedge \varphi(b)$. $\varphi(a \vee b)=S_{(a \vee b)^{\prime}}=S_{a^{\prime}} \vee S_{b^{\prime}}=\varphi(a) \vee \varphi(b), \quad \varphi\left(a^{\prime}\right)=S_{a^{\prime}}=\left(S_{a}\right)^{\prime}=(\varphi(a))^{\prime}$. Clearly $\phi$ is both one-one and onto. Hence $B(A) \cong \mathfrak{B}_{S(A)}$.

In [3] we defined a partial ordering on a C-algebra by $x \leq y$ if and only if $y \wedge x=x$ and we studied the properties of this partial ordering. We gave a number of equivalent conditions in terms of this partial ordering for a $\mathrm{C}$-algebra to become a Boolean algebra. In [4] we proved that, for each $x \in A$, $A_{x}=\{s \in A \mid s \leq x\}$ is itself a C-algebra under induced operations $\wedge, \vee$ and the unary operation is defined by $s^{*}=x \wedge s^{\prime}$ we also observed that $A_{x}$ need not be an algebra of A because the unary operation in $A_{x}$ is not the restriction of the unary operation. For each $x \in A$, we proved that $A_{x}$ is isomorphic to the quotient algebra $A / \theta_{x}$ where $\theta_{x}=\{(p, q) \in A \times A \mid x \wedge p=x \wedge q\}$. We can easily see that the $\mathrm{C}$-algebras $S_{x}, A_{x}$ are different in general where $x \in A$.

Now, we prove that the set of all $A_{a}$ 's where $a \in B(A)$ is a Boolean algebra under set inclusion. The following theorem can be proved analogous to Theorem 4.6.

Theorem 4.8. Let A be a C-algebra with T. Then $\mathfrak{B}_{R(A)}:=\left\{A_{a} \mid a \in B(A)\right\}$ is a Boolean Algebra under set inclusion in which the supremum of $\left\{A_{a}, A_{b}\right\}=A_{a \vee b}$ and the infimum of $\left\{A_{a}, A_{b}\right\}=A_{a \wedge b}$.

The proof of the following theorem is analogous to that of Theorem 4.7.

Theorem 4.9. Let $\mathrm{A}$ be a C-algebra with $\mathrm{T}$ Define $f: B(A) \rightarrow \mathfrak{B}_{R(A)}$ by $f(a)=A_{a}$ for all $a \in B(A)$. Then $f$ is an isomorphism.

The following corollary can be proved directly from Theorems 4.7 and 4.9. 
Corollary 4.10. Let $\mathrm{A}$ be a $\mathrm{C}$-algebra with $\mathrm{T}$. Then $\mathfrak{B}_{R(A)}, B(A)$ and $\mathfrak{B}_{S(A)}$ are isomorphic to each other.

\section{References}

[1] Guzman, F. \& Squier, C., The Algebra of Conditional Logic, Algebra Universalis, 27, pp. 88-110, 1990.

[2] Swamy, U.M., Rao, G.C., Sundarayya, P. \& Kalesha Vali, S., Semilattice Structures on a C-algebra, Southeast Asian Bulletin of Mathematics, 33, pp. 551-561, 2009.

[3] Rao, G.C., Sundarayya, P., C-algebra as a Poset, International Journal of Mathematical Sciences, Serials Pub., New Delhi, 4(2), pp. 225-236, Dec. 2005.

[4] Rao, G.C., Sundarayya, P., Decompositions of a C-algebra, International Journal of Mathematics and Mathematical Sciences, Hindawi Pub. Cor. U.S.A., 2006(3), Article ID 78981, pp. 1-8, 2006.

[5] Swamy, U.M., Rao,G.C. \& Ravi Kumar, R.V.G., Centre of a C-algebra, Southeast Asian Bulletin of Mathematics, 27, pp. 357-368, 2003.

[6] Stanely, B. \& Sankappanavar, H.P., A Course in Universal Algebra, Springer Verlag, 1981. 